\title{
Instrumentation for High Resolution Tomography for Characterization of Microstructures in Light-weight Alloys
}

\author{
Michael Salamon ${ }^{1}$, Norman Uhlmann ${ }^{1}$ Nicole Frindt ${ }^{2}$, Dirk Steiner ${ }^{2}$ and Thomas Wenzel ${ }^{2}$ \\ 1. Fraunhofer EZRT, Fürth, Germany \\ 2. YXLON International GmbH, Hamburg, Germany
}

Present methods for characterization of microstructures in alloys like the analysis of different material phases, dendrites, grain sizes and boundaries are mainly based on the 2D examination of grinded and polished surfaces. In order to perform a 3D analysis a large effort of preparation in multiple grinding and polishing is necessary and finally leads to a destruction of the specimen. In addition to the present used methods, highest resolution X-ray computed tomography (Sub $\mu-C T)$ can offer additional value. Sub $\mu$ $\mathrm{CT}$ is not only able to create a whole $3 \mathrm{D}$ sample volume for investigations but is also able to create slices of a specimen at any needed position and orientation in the volume without destroying the object and area of interest. Additionally the effort for sample preparation is reduced to a minimum compared with other investigation methods. These added values for the field of material characterization motivated research and development in the field of X-ray instrumentation in the past years.

Latest developments offer the possibility to visualize structures in the magnitude of a few micrometers. In order to create comparable results to standard methods, the X-ray instrumentation has to meet certain requirements. Beside the spatial resolution achievable in a single radiographic image using microfocus $\mathrm{X}$-ray sources, the long term stability of the X-ray equipment and the measured object itself plays a major role in Sub $\mu-\mathrm{CT}$. During the relatively long scan periods of several hours resulting from the focal spot to flux ratio, the only movement allowed, is the precise rotation of the object. Furthermore the samples shape and size itself, as also the adjusted spectra and contrast sensitivity of the detector is significant for the detectability of finest contrasts and structures differences inside the sample. Considering these circumstances the Sub- $\mu$ CT can extend the standard metallographic methods by a powerful and lean-preparation 3D method. Even thus it might appear that the computed tomography could already replace standard metallographic investigation methods due to its high potential in terms of 3D imaging, there are limitations resulting on the one hand from the radiation physics themselves and on the other hand from the applied standards and procedures established by 2D examination. Especially for microstructural features like porosities shown in Figure 1 the quantities measured in 2D and 3D results can vary in the order of a magnitude. Adapting the quantification methods and procedures to the volume data type the quality of the metallographic results can be significantly enhanced, empowering the material science.

To reach the requirement for Sub $\mu$-CT 3D sample volumes with a resolution of a few micrometers we used the latest generation of YXLON microfocus X-ray sources for our investigations. The YXLON microfocus source FXT190.61, which is embedded into the YXLON CT systems FF20 and FF35 has a very robust longtime stability. This results in an image shift of less than $3 \mu \mathrm{m}$ and thereby to a very stable image contrast during an operation time of more than 7 hours. Measurements of this very stable image contrast are shown in figure 2. In addition this microfocus X-ray source shows a very high detail detectability of $150 \mathrm{~nm}$, which is demonstrated in figure $3 \mathrm{a}$ ) and b). In this contribution we present the influence of different effects, like drift of the focal spot, on the quality and robustness of high resolution CT scans. Furthermore we show an instrumentation, which provides the necessary long term stability to 
produce high contrast and high resolution 3D metallographic evaluation. Then we focus on the comparison of standard metallographic methods with the CT investigation. As a representative for the automotive industry an Al-Si alloy with $\mathrm{Fe}$ and $\mathrm{Cu}$ microstructures (figure $4 \mathrm{c}$ and $\mathrm{d}$ ) is used to demonstrate the possibilities and limitations of the computed tomography method. Additionally other applications and examples that benefit from the non-destructive 3D method will be discussed.

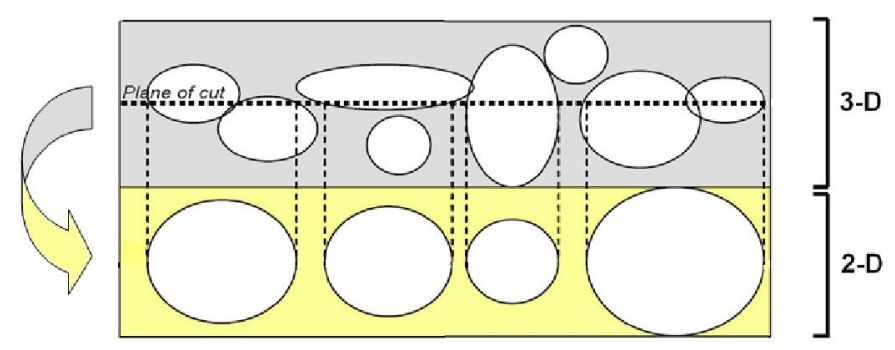

Figure 1. Difficulty of the comparison between the 2D and 3D results. The depth information of a pore is not available from the single cross-section analysis.
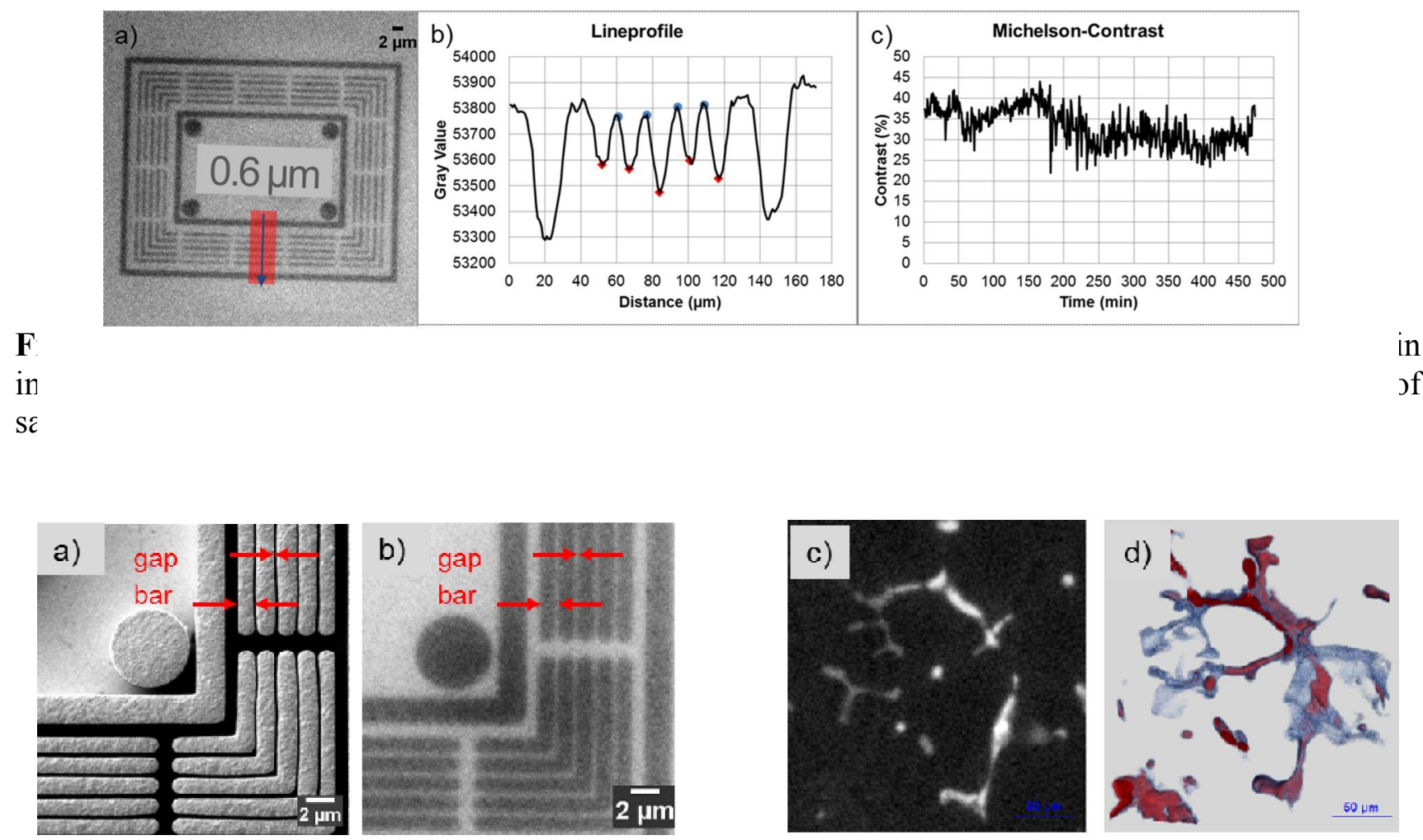

Figure 3. a) SEM image of a sample with bar distance $1160 \mathrm{~nm}$ and gap distance $150 \mathrm{~nm}$. b) 2D X-Ray image of same sample demonstrating the detail detectability of $150 \mathrm{~nm}$ with YXLON FXT190.61 at 160 $\mathrm{kV}$. c) 2D cross section and d) 3D rendering of $\mathrm{Fe}$ and $\mathrm{Cu}$ microstructures in AlSi alloy. 